\title{
Quantum effects of a massive 3-form coupled to a Dirac field.
}

\author{
Antonio Aurilia* \\ Department of Physics, California State Polytechnic University-Pomona \\ Euro Spallucc $\dagger$ \\ Dipartimento di Fisica Teorica, Università di Trieste and INFN, Sezione di Trieste
}

(Dated: June 24, 2018)

\begin{abstract}
The computation of the quantum vacuum pressure must take into account the contribution of zeropoint oscillations of a rank-three gauge field $A_{\mu \nu \rho}$. This result was established in a previous paper where we calculated both the Casimir pressure within a region of vacuum simulating a hadronic bag and the Wilson factor for the three-index potential associated with the boundary of the bag. The resulting "volume law" satisfied by the Wilson loop is consistent with the basic confining requirement that the static inter-quark potential increases with the distance between two test charges. As a sequel to that paper, we consider here the coupling of $A_{\mu \nu \rho}$ to the generic current of a matter field, later identified with the spin density current of a Dirac field. In fact, one of the objectives of this paper is to investigate the impact of the quantum fluctuations of $A_{\mu \nu \rho}$ on the effective dynamics of the spinor field. The consistency of the field equations, even at the classical level, requires the introduction of a mass term for $A_{\mu \nu \rho}$. In this case, the Casimir vacuum pressure includes a contribution that is explicitly dependent on the mass of $A_{\mu \nu \rho}$ and leads us to conclude that the mass term plays the same role as the infrared cutoff needed to regularize the finite volume partition functional previously calculated in the massless case. Remarkably, even in the presence of a mass term, $A_{\mu \nu \rho}$ contains a mixture of massless and massive spin- 0 fields so that the resulting equation is still gauge invariant. This is yet another peculiar, but physically relevant property of $A_{\mu \nu \rho}$ since it is reflected in the effective dynamics of the spinor fields and confirms the confining property of $A_{\mu \nu \rho}$ already expected from the earlier calculation of the Wilson loop.
\end{abstract}

\section{INTRODUCTION}

This the third in a series of articles devoted to an in-depth study of the classical and quantum properties of a rank-three gauge form $A_{\mu \nu \rho}$ in four spacetime dimensions [1], 2].

In a previous paper, hereafter referred to as II [2], we have calculated the contribution to the vacuum pressure, or Casimir energy density, due to the quantum fluctuations of a massless antisymmetric tensor gauge field $A_{\mu \nu \rho}$ with an associated field strength

$$
F_{\lambda \mu \nu \rho}=\partial_{[\lambda} A_{\mu \nu \rho]}
$$

that represents, classically, a non-vanishing constant background field.

Our motivation for investigating the quantum properties of the generalized Maxwell field (1I) was discussed in II. Here, we simply recall that the $F$-field may be related to two central issues in modern theoretical physics, namely, i) the problem of dark matter/energy in the universe via the cosmological constant [1], [3], [4] and ii) the outstanding problem of color confinement in $Q C D$ via the " bag constant " [5], [6]. Both constants, cosmological and hadronic, can be effectively described by the $A_{\mu \nu \rho}$ field.

Apart from these physical considerations, the $F$-field is intriguing because of its deceptive simplicity. Here we have essentially a "constant" disguised as a gauge field, a situation all too familiar from the study of "electrodynamics" in two spacetime dimensions [7]. In actual fact, a study of $A_{\mu \nu \rho}$ is a study of the quantum vacuum and some caution should be exercised before dismissing it. Thus, a consistent quantum formulation of this constant gauge field requires a modification of the "sum over histories approach" in the sense that the path integral must take into account the whole family of constant field configurations having support only over a spacetime region with a finite volume $V$

$$
\int[D F] \delta\left[\partial_{\lambda} F^{\lambda \mu \nu \rho}\right] \exp \left(-\frac{1}{2 \times 4 !} \int_{V} d^{4} x F^{\lambda \mu \nu \rho} F_{\lambda \mu \nu \rho}\right) \propto \int_{-\infty}^{\infty} d f \exp \left(-\frac{f^{2}}{2} V\right)
$$

\footnotetext{
*Electronic address: aaurilia@csupomona.edu

${ }^{\dagger}$ Electronic address: spallucci@trieste.infn.it
} 
where $f$ is an arbitrary constant labeling each classical solution of the free field equations

$$
\partial_{\lambda} F^{\lambda \mu \nu \rho}=0
$$

While the generalized Maxwell field (1) propagates no physical quanta, it gives rise to a static effect, namely, a Casimir vacuum pressure that is inversely proportional to the volume of quantization $V$. This result was confirmed by an explicit calculation of the vacuum expectation value of the energy-momentum tensor associated with the $F$-field

$$
\left.\left\langle T_{\mu \nu}\right\rangle\right|_{g=\delta}=\left\langle\frac{1}{3 !} F_{\mu \rho \sigma \tau} F_{\nu}^{\rho \sigma \tau}-\frac{1}{2 \times 4 !} \delta_{\mu \nu} F^{\rho \sigma \tau \lambda} F_{\rho \sigma \tau \lambda}\right\rangle=\delta_{\mu \nu}\left(\rho_{0}+\frac{1}{2 V}\right)
$$

In the above expression, $\rho_{0}$ represents a classical contribution to the vacuum energy and plays the role of an infrared cutoff that is necessary in order to regularize the large volume behavior of the finite volume partition function $Z(V)$ that was explicitly derived in II.

With hindsight, we recognize that the role of the infrared cutoff $\rho_{0}$ may be played by a mass term in the lagrangian for the $F$-field. In the first order formalism this lagrangian reads

$$
L_{0}=\frac{1}{2 \times 4 !} F^{\rho \sigma \tau \lambda} F_{\rho \sigma \tau \lambda}-\frac{1}{4 !} F^{\lambda \mu \nu \rho} \partial_{[\lambda} A_{\mu \nu \rho]}-\frac{m_{A}^{2}}{2 \times 3 !} A^{\mu \nu \rho} A_{\mu \nu \rho} .
$$

In spite of its unusual appearance, the above expression is a Proca-type lagrangian that describes a massive spin-0 field [8], with some qualifications. Indeed, the field equations are

$$
\begin{aligned}
& F_{\lambda \mu \nu \rho}=\partial_{[\lambda} A_{\mu \nu \rho]}, \\
& \partial_{\lambda} F^{\lambda \mu \nu \rho}-m_{A}^{2} A^{\mu \nu \rho}=0 .
\end{aligned}
$$

From Eq.(7) follows the constraint equation

$$
\partial_{\lambda} A^{\lambda \mu \nu}=0
$$

due to the antisymmetry of $F_{\lambda \mu \nu \rho}$. Substituting equations (6) and (8) into Eq.(7) we arrive at the Klein-Gordon equation

$$
\left(-\partial^{2}+m_{A}^{2}\right) A^{\lambda \mu \nu}=0 .
$$

On the other hand, the constraint (8) implies that

$$
A^{\lambda \mu \nu}=\frac{1}{m_{A}} \epsilon^{\lambda \mu \nu \rho} \partial_{\rho} \phi
$$

so that the scalar field $\phi$ satisfies the equation

$$
\left(-\partial^{2}+m_{A}^{2}\right)\left(-\partial^{2}\right) \phi=0 .
$$

Several observations are in order here. First, it should be noted that in the massless case the Maxwell field strength possesses no physical degrees of freedom in view of its invariance under the tensor gauge transformation

$$
\delta A_{\mu \nu \rho}=\partial_{[\mu} \lambda_{\nu \rho]} .
$$

In this connection, it is also worth observing that the Proca equation for $A_{\mu \nu \rho}$ leads to a gauge invariant equation in spite of the presence of a mass term. This is implicit in the form of Eq. (11) due to the presence of the extra d'Alembertian. Alternatively, in terms of the dual field 


$$
{ }^{*} A^{\mu} \equiv \frac{1}{3 !} \epsilon^{\mu \nu \rho \sigma} A_{\nu \rho \sigma}=\frac{1}{m_{A}} \partial^{\mu} \phi
$$

it follows from Eq.(9) that

$$
\left(-\partial^{2}+m_{A}^{2}\right) \partial_{\mu}{ }^{*} A^{\mu}=0
$$

which is invariant under the dual gauge transformation

$$
\delta^{*} A^{\mu}=\frac{1}{3 !} \epsilon^{\mu \nu \rho \sigma} \partial_{[\nu} \lambda_{\rho \sigma]} .
$$

The advantage of using the first order formalism in the path integral method as a means of getting around the lengthy Fadeev-Popov procedure [9] necessary to eliminate those spurious degrees of freedom in the massless case was discussed in II. We note, incidentally, that the presence of the extra D' Alambertian in the massive case may be construed as further evidence of the confining properties of the $A_{\mu \nu \rho}$-field already expected from the earlier calculation of the Wilson loop [2].

A general solution of the field equations, in the first order formulation, can be obtained after combining Eq.(7) and Eq.(10) into the single equation

$$
\partial_{\lambda} F^{\lambda \mu \nu \rho}=m_{A} \epsilon^{\mu \nu \rho \sigma} \partial_{\sigma} \phi
$$

In Eq. (16) the mass term plays the role of a source term for the Maxwell field. Accordingly, a formal expression for $F^{\lambda \mu \nu \rho}$ is as follows

$$
F^{\lambda \mu \nu \rho}=\epsilon^{\lambda \mu \nu \rho} f-m_{A} \partial^{[\lambda} \frac{1}{-\partial^{2}} \epsilon^{\mu \nu \rho] \tau} \partial_{\tau} \phi
$$

where $f$ represents an arbitrary ( constant ) solution of the associated homogeneous equation. It is precisely this constant background field $f$ that gives rise to the Casimir effect that we have investigated in II and summarized at the beginning of this introduction.

Against this background, the purpose of this paper is twofold. First, we wish to investigate the contribution to the vacuum pressure due to the quantum fluctuations of the massive three-index field $A_{\mu \nu \rho}$. Our second objective is to investigate the effective theory of a spinor field confined to a volume of quantization under the vacuum pressure generated by the zero-point oscillations of $A_{\mu \nu \rho}$.

As anticipated earlier, we find the same volume dependence of the vacuum pressure as in the massless case, plus an extra contribution that depends explicitly on the mass of $A_{\mu \nu \rho}$ and plays the same role of the phenomenological parameter $\rho_{0}$ reported in Eq.(4). These results are obtained in Sect.2.

Section.3 is devoted to the calculation of the finite volume effective action of a spinor field. Our motivation is that the gauge invariance of the $A_{\mu \nu \rho}$ field in the massless case requires that its classical matter counterpart be given by the three-dimensional world history of a relativistic membrane. At the quantum level this coupling may be naturally realized through the spin-density current of a Dirac field. Our overall approach is nonperturbative to the extent that we sum over all possible configurations of $A_{\mu \nu \rho}$ in order to determine its quantum effects on the effective dynamics of the spinor field. Section. 4 concludes the paper with a summary of our results and an outlook of further research into the quantum properties of the $A_{\mu \nu \rho}$ field.

\section{VACUUM FLUCTUATIONS OF THE MASSIVE $A_{\mu \nu \rho}$ FIELD.}

We have seen in the Introduction that there is a discontinuity between the massless and massive case in the field equation for $A_{\mu \nu \rho}$. In the massless case, there are no radiative degrees of freedom and the field strength describes a non-vanishing constant background field within a finite volume. There are, however, quantum vacuum fluctuations associated even with a constant gauge field and the corresponding vacuum energy/pressure is explicitly given by Eq. (4) that was derived in II. In the massive case, on the other hand, there are physical quanta of spin-0 associated with $A_{\mu \nu \rho}$. It seems reasonable, therefore, to expect an additional contribution to the Casimir energy density/pressure related to the mass of $A_{\mu \nu \rho}$. That this is the case can be seen by calculating the finite volume partition functional 


$$
Z(V)=\int[D F][D A] \delta\left[\partial_{\mu} A^{\mu \nu \rho}\right] \exp \left(-\int d^{4} x\left[\frac{1}{2 \times 4 !} F^{\rho \sigma \tau \lambda} F_{\rho \sigma \tau \lambda}-\frac{1}{4 !} F^{\lambda \mu \nu \rho} \partial_{[\lambda} A_{\mu \nu \rho]}-\frac{m_{A}^{2}}{2 \times 3 !} A^{\mu \nu \rho} A_{\mu \nu \rho}\right]\right)
$$

Note that the expression (18) explicitly takes into account the constraint equation (8).

Let us start the computation of $Z(V)$ from the $A$-integration. Taking into account Eq.(10), which is equivalent to the constraint (8), we find

$$
Z(V)=\int[D F][D \phi] \exp \left(-\int d^{4} x\left[\frac{1}{2 \times 4 !} F^{\rho \sigma \tau \lambda} F_{\rho \sigma \tau \lambda}+\frac{1}{3 !}\left(\partial_{\lambda} F^{\lambda \mu \nu \rho}\right) \epsilon_{\mu \nu \rho \sigma} \partial^{\sigma} \phi-\frac{m_{A}^{2}}{2} \phi^{2}\right]\right)
$$

The form of Eq.(19) suggests that it may be convenient to split the $F$ - field in the sum of two parts $\widehat{F}^{\lambda \mu \nu \rho}$ and $\widetilde{F}^{\lambda \mu \nu \rho}$ such that

$$
\begin{aligned}
& F^{\lambda \mu \nu \rho}=\widehat{F}^{\lambda \mu \nu \rho}+\widetilde{F}^{\lambda \mu \nu \rho} \\
& \partial_{\lambda} \widehat{F}^{\lambda \mu \nu \rho}=0, \\
& \partial_{\lambda} \widetilde{F}^{\lambda \mu \nu \rho} \neq 0 \equiv h^{\mu \nu \rho}, \quad \partial_{\mu} h^{\mu \nu \rho}=0 .
\end{aligned}
$$

Accordingly, the functional integration over $F$ is factorized into an integration over divergence free paths times an integration over remaining histories parametrized by $h^{\mu \nu \rho}$. The latter integration is restricted to the subspace of divergence free $h$-configurations by the consistency condition (22)

$$
[D F]=[D \widehat{F}] \delta\left[\partial_{\lambda} \widehat{F}^{\lambda \mu \nu \rho}\right][D \widetilde{F}][D h] \delta\left[\partial_{\lambda} \widetilde{F}^{\lambda \mu \nu \rho}-h^{\mu \nu \rho}\right] \delta\left[\partial_{\mu} h^{\mu \nu \rho}\right]
$$

Furthermore, Eq.21 implies that the corresponding functional measure in (23) must involve only constant field configurations, namely, arbitrary constant solutions $f$ of Eq.(21) with dimensions $[f]=$ mass $^{2}$,

$$
[D \widehat{F}] \delta\left[\partial_{\lambda} \widehat{F}^{\lambda \mu \nu \rho}\right] \propto d f[D \widehat{F}] \delta\left[\widehat{F}^{\lambda \mu \nu \rho}-f \epsilon^{\lambda \mu \nu \rho}\right] .
$$

Therefore, integrating over $\widehat{F}$ means summing over all constant $\widehat{F}$-field configurations that satisfy the constraint (21). It seems worth observing, on the technical side, that this procedure of integration is identical to that introduced by the authors in the case of a free point particle in which the integration over the momentum trajectories is constrained to satisfy the classical equations of motion $\dot{q}^{i}(t)=0[10]$. In both cases, the functional integration is reduced to an ordinary integration, namely, $[D q] \delta\left[\dot{q}^{i}(t)\right] \longrightarrow d^{3} q /(2 \pi)^{3}$. However, by extracting an ordinary differential $d f$ out of the dimensionless functional measure $[D F]$ we have to rescale the sum against an arbitrarily fixed standard measure $m_{F}^{2}$ with dimensions of a mass squared.

Taking all of the above into account, the partition functional becomes

$$
\begin{aligned}
& Z(V)=\frac{1}{m_{F}^{2}} \int_{-\infty}^{\infty} d f \exp \left(-\frac{f^{2}}{2} V\right) \int[D \phi]\left[D h^{\mu \nu \rho}\right] \delta\left[\partial_{\mu} h^{\mu \nu \rho}\right] \exp \left(-S\left[h^{\mu \nu \rho}, \phi\right]\right) \\
& S\left[h^{\mu \nu \rho}, \phi\right]=\int d^{4} x\left[-\frac{1}{2 \times 3 !} h^{\mu \nu \rho} \frac{1}{-\partial^{2}} h_{\mu \nu \rho}+\frac{1}{4 !} \phi \epsilon_{\lambda \mu \nu \rho} \partial^{[\lambda} h^{\mu \nu \rho]}-\frac{m_{A}^{2}}{2}(\partial \phi)^{2}\right] .
\end{aligned}
$$

Since $h^{\mu \nu \rho}$ is divergenceless by definition, as seen in Eq.(22), our next step is to rewrite it as the Hodge dual of a longitudinal four-vector:

$$
h^{\mu \nu \rho}=\epsilon^{\mu \nu \rho \sigma} \partial_{\sigma} \xi
$$

Then, integrating over $f$ and $\xi$ gives

$$
\begin{aligned}
Z(V) & =\left(\frac{2 \pi}{V m_{F}^{4}}\right)^{1 / 2} \int[D \phi][D \xi] \exp \left(-\int d^{4} x\left[\frac{1}{2} \partial_{\mu} \xi \frac{1}{-\partial^{2}} \partial^{\mu} \xi+\frac{m_{A}^{2}}{2}\left(\partial^{2} \xi\right)(\partial \phi)^{2}\right]\right) \\
& =\left(\frac{2 \pi}{V m_{F}^{4}}\right)^{1 / 2} \int[D \phi] \exp \left(-\int d^{4} x\left[\frac{1}{2} \phi\left(-\partial^{2}+m_{A}^{2}\right)\left(-\partial^{2}\right) \phi\right]\right) .
\end{aligned}
$$


Note that this round of calculations has led us to a type of scalar field equation in the expression (28) that is precisely of the form given in Eq. (11) discussed in the Introduction. Note, in particular, the characteristic presence of the double d'Alembertian that we have linked to the presence of a gauge invariant mass term. The remaining integration over $\phi$ is gaussian and leads to the formal result

$$
\begin{aligned}
Z(V) & \equiv\left(\frac{2 \pi}{V m_{F}^{4}}\right)^{1 / 2} \exp [-W(V)] \\
& =\left(\frac{2 \pi}{V m_{F}^{4}}\right)^{1 / 2}\left[\operatorname{det}\left(-\partial^{2}\right)\left(-\partial^{2}+m_{A}^{2}\right)\right]^{-1 / 2}
\end{aligned}
$$

In order to calculate the above determinant in closed form, the usual procedure is to perform the calculation in the large volume limit, namely, $V m_{A}^{4}>>1$. In this limit, the spectrum of the operator $-\partial^{2}+m_{A}^{2}$ can be treated as a continuum so that the sum over the eigenvalues can be approximated by the following integral

$$
\begin{aligned}
W(V) & =\frac{1}{2} V \int \frac{d^{4} k}{(2 \pi)^{4}}\left[\ln \left(k^{2}+m_{A}^{2}\right)+\ln k^{2}\right] \\
& =-\frac{1}{2} V \int \frac{d^{4} k}{(2 \pi)^{4}} \int_{0}^{\infty} \frac{d \tau}{\tau}\left[\exp \left[-\tau\left(k^{2}+m_{A}^{2}\right)\right]+\exp \left(-\tau k^{2}\right)\right]
\end{aligned}
$$

Let us consider first the mass dependent term. We will see at the end of this calculation that the finite part contributing to $W(V)$ is actually proportional to $m_{A}^{4}$. Anticipating this result we drop altogether the contribution coming from $\ln k^{2}$ in the above expression. The expression (30) is divergent in the limit $\tau \rightarrow 0$, i.e., in the shortdistance regime. Therefore, it is convenient to use the proper time regularization procedure to cast Eq.(30) in the following form

$$
W_{\epsilon}(V)=-\frac{1}{2} \frac{1}{(2 \pi)^{2}} V \mu^{-2 \epsilon} \int_{0}^{\infty} \frac{d \tau}{\tau^{3+\epsilon}} \exp \left(-\tau m_{A}^{2}\right)
$$

where we have introduced a mass parameter $\mu$ so that $W$ is a-dimensional. By rescaling the proper time $x \equiv \tau m_{A}^{2}$, we obtain

$$
\begin{aligned}
W_{\epsilon}(V) & =-\frac{1}{2} \frac{1}{(2 \pi)^{2}} V m_{A}^{4}\left(\frac{\mu^{2}}{m_{A}^{2}}\right)^{-\epsilon} \int_{0}^{\infty} d x x^{-3-\epsilon} \exp (-x) \\
& =-\frac{1}{2} \frac{1}{(2 \pi)^{2}} V m_{A}^{4}\left(\frac{\mu^{2}}{m_{A}^{2}}\right)^{-\epsilon} \Gamma(-2-\epsilon)
\end{aligned}
$$

where

$$
\begin{aligned}
\Gamma(-2-\epsilon) & =-\frac{1}{\epsilon(1+\epsilon)(2+\epsilon)} \Gamma(1+\epsilon) \\
& \approx-\frac{1}{2 \epsilon}\left[1-\left(\gamma+\frac{3}{2}\right) \epsilon+\ldots\right] \\
\gamma & \equiv-\Gamma^{\prime}(0)=\quad \text { Euler Constant }
\end{aligned}
$$

and the expression in Eq. (34) refers to the limit $\epsilon \rightarrow 0$. From here we isolate the pole singularity from the convergent part of the integral as follows

$$
\begin{aligned}
W_{\epsilon}(V) & \equiv W_{\infty}+W_{\text {Reg. }} \\
W_{\infty} & \equiv \frac{1}{4 \epsilon} \frac{1}{(2 \pi)^{2}} V m_{A}^{4} \\
W_{\text {Reg. }} & \equiv-\frac{1}{4} \frac{1}{(2 \pi)^{2}} V m_{A}^{4}\left[\ln \left(\frac{\mu^{2}}{m_{A}^{2}}\right)+\gamma+\frac{3}{2}\right] .
\end{aligned}
$$


Then, in the minimal subtraction scheme we obtain:

$$
\ln Z_{\text {Reg. }}=-\frac{1}{2} \ln \frac{V m_{A}^{4}}{2 \pi}-\frac{1}{4} \frac{1}{(2 \pi)^{2}} V m_{A}^{4}\left[\ln \left(\frac{m_{A}^{2}}{\mu^{2}}\right)-\gamma-\frac{3}{2}\right] .
$$

With this result in hands we are finally able to calculate the vacuum pressure as follows

$$
p \equiv-\frac{\partial}{\partial V} \ln Z_{\text {Reg. }}=\frac{1}{2 V}+\frac{1}{4} \frac{1}{(2 \pi)^{2}} m_{A}^{4}\left[\ln \left(\frac{m_{A}^{2}}{\mu^{2}}\right)-\gamma-\frac{3}{2}\right] .
$$

This result should be compared with equation (4) in the Introduction. Equation(4) represents the outcome of our calculation in the massless case discussed in II . The first term in Eq. (40) represents the Casimir contribution which dominates for small volumes and agrees with the previous result for the massless case. The second term represents a new contribution that dominates in the large volume limit and corresponds to the term $\rho_{0}$ that appears in Eq. (44). This comparison gives an explicit expression for $\rho_{0}$ in terms of the $A_{\mu \nu \rho}$ mass $m_{A}$ and regulator mass $\mu$.

\section{THE INTERACTING CASE.}

In our previous paper, II, we have calculated the Wilson loop for the three-index potential associated with a bag with a boundary represented by the three-dimensional world history of a closed spherical membrane. From the Wilson loop we derived the static potential between two opposite points on the surface of the bag and found it proportional to the volume enclosed by the surface. This "volume law" seems a natural extension of the area law found in the more conventional case of quantum chromodynamic strings and is consistent with the basic underlying idea of confinement that it requires an infinite amount of energy to separate the two points [11].

It is against this background computation that we wish to consider the coupling of $A_{\mu \nu \rho}$ to a current $J^{\mu \nu \rho}$ that is later identified with the spin-density current of a Dirac field.

Mathematically, this new situation amounts to taking as a new action

$$
S=\int d^{4} x\left[\frac{1}{2 \times 4 !} F^{\rho \sigma \tau \lambda} F_{\rho \sigma \tau \lambda}-\frac{1}{4 !} F^{\lambda \mu \nu \rho} \partial_{[\lambda} A_{\mu \nu \rho]}-\frac{m_{A}^{2}}{2 \times 3 !} A^{\mu \nu \rho} A_{\mu \nu \rho}-\frac{\kappa}{3 !} A_{\mu \nu \rho} J^{\mu \nu \rho}\right]
$$

from which we find the field equations

$$
\begin{aligned}
& F_{\lambda \mu \nu \rho}=\partial_{[\lambda} A_{\mu \nu \rho]}, \\
& \partial_{\lambda} F^{\lambda \mu \nu \rho}-m_{A}^{2} A^{\mu \nu \rho}=\kappa J^{\mu \nu \rho} .
\end{aligned}
$$

Equation (43) was studied in I where the introduction of a mass term was required by the consistency of the field equations. As a matter of fact, without a mass term Eq.(43) is inconsistent as a consequence of the antisymmetry of $F^{\lambda \mu \nu \rho}$ unless the current on the right side is divergence free. Thus, coupling $A^{\mu \nu \rho}$ to a spinor field requires, in general, the presence of a mass term. From Eq.(43) follows the new constraint equation

$$
\partial_{\lambda} A^{\lambda \mu \nu}=-\frac{\kappa}{m_{A}^{2}} \partial_{\mu} J^{\mu \nu \rho} \equiv-\frac{\kappa}{m_{A}^{2}} j^{\nu \rho}
$$

where we have defined $j^{\nu \rho}$ as the divergence of $J^{\mu \nu \rho}$. A formal solution of the constraint Eq.(44) is

$$
A^{\lambda \mu \nu}=\frac{1}{m_{A}} \epsilon^{\lambda \mu \nu \rho} \partial_{\rho} \phi-\frac{\kappa}{m_{A}^{2}} \partial^{[\lambda} \frac{1}{\partial^{2}} j^{\mu \nu]} .
$$

Substituting Eq.(45) into Eq.(43), we arrive at the following field equation

$$
\partial_{\lambda} F^{\lambda \mu \nu \rho}-m_{A} \epsilon^{\mu \nu \rho \sigma} \partial_{\sigma} \phi=\kappa\left(J^{\mu \nu \rho}-\partial^{[\mu} \frac{1}{\partial^{2}} j^{\nu \rho]}\right) .
$$

Therefore, in analogy to the free field case, Eq.(17), a generic solution of Eq.(46) can be expressed as follows 


$$
\begin{aligned}
F^{\lambda \mu \nu \rho} & =\epsilon^{\lambda \mu \nu \rho} f+m_{A} \partial^{[\lambda} \frac{1}{\partial^{2}} \epsilon^{\mu \nu \rho] \tau} \partial_{\tau} \phi+\kappa\left(J^{\mu \nu \rho}-\partial^{[\mu} \frac{1}{\partial^{2}} j^{\nu \rho]}\right) \\
& \equiv \epsilon^{\lambda \mu \nu \rho} f+m_{A} \partial^{[\lambda} \frac{1}{\partial^{2}} \epsilon^{\mu \nu \rho] \tau} \partial_{\tau} \phi+\kappa \widehat{J}^{\mu \nu \rho}
\end{aligned}
$$

Up until now, the nature of the three index current in the above expressions has not been specified. In our previous papers, both I and II, $J^{\mu \nu \rho}$ was identified with the classical current associated with the world history of a relativistic membrane. Presently, as a stepping stone toward a forthcoming discussion of the quantum chromodynamic case, it is instructive to identify $J^{\mu \nu \rho}$ with the spin-density current of a Dirac field. As a matter of fact, as anticipated in the Introduction, one of the main purposes of this paper is to determine the form of the effective action $\Gamma[\bar{\psi}, \psi]$ for a Dirac field under the influence of the quantum vacuum fluctuations of $A_{\mu \nu \rho}$.

In the massless case, considered in II, this same objective was achieved by an explicit calculation of the Wilson factor defined by

$$
\begin{aligned}
W[J]=\left\langle\exp \left(-\frac{\kappa}{3 !} \int d^{4} x A_{\mu \nu \rho} J^{\mu \nu \rho}\right)\right\rangle & =\frac{Z[J]}{Z[0]} \\
& \equiv \exp (-\Gamma[J])
\end{aligned}
$$

where

$$
J^{\mu \nu \rho}=\int_{\partial B} \delta^{4)}[x-Y] d y^{\mu} \wedge d y^{\nu} \wedge d y^{\rho}
$$

represents the classical current of a relativistic test bubble. Presently we wish to undertake the same calculation in the full quantum case in which the current (49) is replaced by the spin density current of a spinor field in the background vacuum that we have discussed in the previous section

$$
J^{\mu \nu \rho} \longrightarrow \Sigma^{\mu \nu \rho} \equiv \bar{\psi} \gamma^{\mu} \gamma^{\nu} \gamma^{\rho} \psi .
$$

Mathematically, our task is to integrate the partition functional

$$
\begin{aligned}
Z[\Sigma, V]= & \int[D F][D A] \delta\left[m_{A}^{2} \partial_{\mu} A^{\mu \nu \rho}-\kappa \partial_{\mu} \Sigma^{\mu \nu \rho}\right] \times \\
& \exp \left(-\int d^{4} x\left[\frac{1}{2 \times 4 !} F^{\rho \sigma \tau \lambda} F_{\rho \sigma \tau \lambda}-\frac{1}{4 !} F^{\lambda \mu \nu \rho} \partial_{[\lambda} A_{\mu \nu \rho]}-\frac{m_{A}^{2}}{2 \times 3 !} A^{\mu \nu \rho} A_{\mu \nu \rho}-\frac{\kappa}{3 !} \Sigma^{\mu \nu \rho} A_{\mu \nu \rho}\right]\right) .
\end{aligned}
$$

Before proceeding with the calculations, let us introduce the notation

$$
\begin{aligned}
& J^{5 \mu} \equiv \frac{1}{3 !} \epsilon^{\mu}{ }_{\nu \rho \tau} \Sigma^{\mu \nu \rho}, \\
& \left.j^{\nu \rho} \equiv \partial_{\mu} \Sigma^{\mu \nu \rho}=\frac{1}{2} \epsilon^{\mu \nu \rho \tau} \partial_{[\mu} J^{5} \tau\right], \\
& J^{5 \mu} \equiv J^{5 \mu}{ }_{T}+J^{5 \mu}{ }_{L}, \\
& J^{5 \mu}{ }_{T}=\left(\delta^{\mu}{ }_{\nu}-\partial^{\mu} \frac{1}{\partial^{2}} \partial_{\nu}\right) J^{5 \nu} \\
& J^{5 \mu}{ }_{L}=\partial^{\mu} \frac{1}{\partial^{2}} \partial_{\nu} J^{5 \nu} .
\end{aligned}
$$

Once again, we begin the computation of $Z[\Sigma, V]$ with the $A$-integration. The constraint (44) is encoded in the functional Dirac-delta in Eq.(51), and enables us to replace $A$ with the classical solution (45). Thus we obtain

$$
\begin{aligned}
Z[\Sigma, V] & =\int[D F][D \phi] \exp \left(-\int d^{4} x\left[\frac{1}{2 \times 4 !} F^{\rho \sigma \tau \lambda} F_{\rho \sigma \tau \lambda}\right.\right. \\
& +\frac{1}{3 !}\left(\frac{1}{m_{A}} \epsilon_{\mu \nu \rho \sigma} \partial^{\sigma} \phi+\frac{\kappa}{m_{A}^{2}} \partial_{[\mu} \frac{1}{-\partial^{2}} j_{\nu \rho]}\right) \partial_{\lambda} F^{\lambda \mu \nu \rho}-\frac{m_{A}^{2}}{2 \times 3 !}\left(\frac{1}{m_{A}} \epsilon_{\mu \nu \rho \sigma} \partial^{\sigma} \phi+\frac{\kappa}{m_{A}^{2}} \partial_{[\mu} \frac{1}{-\partial^{2}} j_{\nu \rho]}\right)^{2} \\
& \left.\left.-\frac{\kappa}{3 !} \Sigma^{\mu \nu \rho}\left(\frac{1}{m_{A}} \epsilon_{\mu \nu \rho \sigma} \partial^{\sigma} \phi+\frac{\kappa}{m_{A}^{2}} \partial_{[\mu} \frac{1}{-\partial^{2}} j_{\nu \rho]}\right)\right]\right)
\end{aligned}
$$


The integration over $F$ proceeds exactly as in the free case. Incidentally, this is one advantage of the first order formalism in which $F$ and $A$ are treated as independent variables. Therefore

$$
\begin{aligned}
Z[\Sigma, V] & =\left(\frac{2 \pi}{m_{F}^{4} V}\right)^{1 / 2} \int[D \phi][D \xi] \exp \left(-\int d^{4} x\left[\frac{1}{2} \partial_{\mu} \xi \frac{1}{-\partial^{2}} \partial^{\mu} \xi\right.\right. \\
& +\frac{1}{3 !}\left(\frac{1}{m_{A}} \epsilon_{\mu \nu \rho \sigma} \partial^{\sigma} \phi+\frac{\kappa}{m_{A}^{2}} \partial_{[\mu} \frac{1}{-\partial^{2}} j_{\nu \rho]}\right) \epsilon^{\lambda \mu \nu \rho} \partial_{\lambda} \xi-\frac{m_{A}^{2}}{2 \times 3 !}\left(\frac{1}{m_{A}} \epsilon_{\mu \nu \rho \sigma} \partial^{\sigma} \phi+\frac{\kappa}{m_{A}^{2}} \partial_{[\mu} \frac{1}{-\partial^{2}} j_{\nu \rho]}\right)^{2} \\
& \left.\left.-\frac{\kappa}{3 !} \Sigma^{\mu \nu \rho}\left(\frac{1}{m_{A}} \epsilon_{\mu \nu \rho \sigma} \partial^{\sigma} \phi+\frac{\kappa}{m_{A}^{2}} \partial_{[\mu} \frac{1}{-\partial^{2}} j_{\nu \rho]}\right)\right]\right)
\end{aligned}
$$

Next, we proceed with the integration of the $\eta$-field noting that it is decoupled from $j^{\nu \rho}$ since

$$
\int d^{4} x \epsilon^{\lambda \mu \nu \rho}\left(\partial_{\lambda} \xi\right) \partial_{[\mu} \frac{1}{-\partial^{2}} j_{\nu \rho]}=-\int d^{4} x \epsilon^{\lambda \mu \nu \rho} \xi \partial_{[\lambda} \partial_{[\mu} \frac{1}{-\partial^{2}} j_{\nu \rho]} \equiv 0
$$

Collecting our results so far, we find

$$
\begin{aligned}
Z[\Sigma, V] & =\left(\frac{2 \pi}{m_{F}^{4} V}\right)^{1 / 2} \int[D \phi] \exp \left(-\int d^{4} x\left[\frac{1}{2 m_{A}^{2}}\left(-\partial^{2}\right) \phi\left(-\partial^{2}\right) \phi\right.\right. \\
& \left.\left.-\frac{m_{A}^{2}}{2 \times 3 !}\left(\frac{1}{m_{A}} \epsilon_{\mu \nu \rho \sigma} \partial^{\sigma} \phi+\frac{\kappa}{m_{A}^{2}} \partial^{[\mu} \frac{1}{-\partial^{2}} j^{\nu \rho]}\right)^{2}-\frac{\kappa}{3 !} \Sigma^{\mu \nu \rho}\left(\frac{1}{m_{A}} \epsilon_{\mu \nu \rho \sigma} \partial^{\sigma} \phi+\frac{\kappa}{m_{A}^{2}} \partial_{[\mu} \frac{1}{-\partial^{2}} j_{\nu \rho]}\right)\right]\right)
\end{aligned}
$$

Before integrating over $\phi$, it is convenient to collect all the terms explicitly depending on $\phi$ and rewrite $Z[\Sigma, V]$ as follows

$$
\begin{aligned}
Z[\Sigma, V]= & \left(\frac{2 \pi}{m_{F}^{4} V}\right)^{1 / 2} \exp \left(-\frac{\kappa^{2}}{2 m_{A}^{2}} \int d^{4} x j^{\nu \rho} \frac{1}{-\partial^{2}} j_{\nu \rho}\right) \times \\
& \int[D \phi] \exp \left(-\int d^{4} x\left[\frac{1}{2 m_{A}^{2}}\left(-\partial^{2}\right) \phi\left(-\partial^{2}\right) \phi+\frac{\kappa}{m_{A}} \phi \partial_{\mu} J^{5 \mu}\right]\right) .
\end{aligned}
$$

The final integration over $\phi$ now leads to the following expression

$$
\begin{aligned}
Z[\Sigma, V] & =\left(\frac{2 \pi}{m_{F}^{4} V}\right)^{1 / 2}\left[\left(-\partial^{2}\right)\left(-\partial^{2}+m_{A}^{2}\right)\right]^{1 / 2} \times \\
& \exp \left(-\frac{\kappa^{2}}{2} \int d^{4} x\left[j^{\nu \rho} \frac{1}{-\partial^{2}} j_{\nu \rho}-\left(\partial_{\mu} J^{5 \mu}\right) \frac{1}{\left(-\partial^{2}\right)\left(-\partial^{2}+m_{A}^{2}\right)}\left(\partial_{\nu} J^{5 \nu}\right)\right]\right) \\
& =\sqrt{\frac{2 \pi}{m_{F}^{4} V}\left[\left(-\partial^{2}\right)\left(-\partial^{2}+m_{A}^{2}\right)\right]^{1 / 2} \times} \\
& \exp \left(-\frac{\kappa^{2}}{2} \int d^{4} x\left[\partial^{[\mu} j_{T}^{5 \nu]} \frac{1}{-\partial^{2}} \partial_{[\mu} j_{T \nu]}^{5}-J_{L}^{5 \nu} \frac{1}{\left(-\partial^{2}\right)\left(-\partial^{2}+m_{A}^{2}\right)} J_{L \nu}^{5}\right]\right) .
\end{aligned}
$$

From here we extract our final result: according to the definition (48) the dynamics of the fermion field is governed by the effective action induced by quantum fluctuations of the $A$-field,

$$
\Gamma[\bar{\psi}, \psi]=\frac{\kappa^{2}}{2} \int d^{4} x\left[\partial^{[\mu} j_{T}^{5 \nu]} \frac{1}{-\partial^{2}} \partial_{[\mu} j_{T \nu]}^{5}-J_{L}^{5 \nu} \frac{1}{\left(-\partial^{2}\right)\left(-\partial^{2}+m_{A}^{2}\right)} J_{L \nu}^{5}\right]
$$

A cursory inspection of the above expression indicates that the original interaction of the $A_{\mu \nu \rho}$ field coupled to the spin-density current corresponds to an effective four-fermion interaction where two distinct components can be identified. One is a long-range interaction that involves only the transverse part of the axial current, the other is a short-range interaction that involves only the longitudinal part of the axial current. This dynamical splitting seems 
noteworthy to the extent that the Green function of the longitudinal component corresponds, once again, to the dipole operator of the scalar field equation (11) derived in the Introduction. To our mind, this suggests that the longitudinal component of the axial current behaves as a spin-0 field which we interpret as a collective excitation, or bound state, generated by the underlying dynamics of the spinor field. According to our line of reasoning, this effect, which one may call dynamical bosonization, should be traced back to the properties of the quantum vacuum created by the $A_{\mu \nu \rho}$ field.

\section{SUMMARY AND OUTLOOK}

In this paper we have resumed an in-depth study of the properties of a rank three, antisymmetric tensor gauge field $A_{\mu \nu \rho}$.

The classical properties of this field, and its counterpart in any number of spacetime dimensions, have been known for a long time [12]. Unlike its electromagnetic counterpart in four dimensions, $A_{\mu \nu \rho}$ does not radiate photons, or any other type of physical wave. However, very much like its "electromagnetic" counterpart in two dimensions, it simply represents a constant background field. Thus, in the free case and in flat spacetime ( of infinite extension ) $A_{\mu \nu \rho}$ cannot be distinguished from the classical "vacuum ". However, dismissing this field as physically irrelevant on this basis, would be too hasty. Indeed, the form of the gauge transformation (12) dictates that $A_{\mu \nu \rho}$ couples to extended objects represented by the world history of relativistic membranes. Furthermore, even in the absence of interaction but in the presence of a mass term, $A_{\mu \nu \rho}$ "comes alive" in the sense that it represents a spin-0 field that obeys a Proca-type equation of the kind (6), (7) discussed in the Introduction. The presence of a gauge invariant mass term was linked in I to the Stueckelberg mechanism for $A_{\mu \nu \rho}$ [13] and shown to be related to the production of dark energy/matter in the universe. The strong formal analogy between this peculiar mechanism of mass generation in four dimensions and a similar mechanism in two dimensions was also emphasized in I.

To our mind, these unique properties of the $A$-field suggest a rather fitting model, at least qualitatively, of a hadronic bag in which the role of the phenomenological "bag constant" is taken over by the infrared cutoff that is necessary to regularize the partition functional that describes the quantum dynamics of $A_{\mu \nu \rho}$. In support of this interpretation, it was necessary to show that there exists a confining potential within the bag. As a matter of fact, an explicit calculation of the Wilson loop associated with a relativistic test bubble simulating the surface of the bag, shows that the static potential between any pair of diametrically opposite points on the surface of the bag is proportional to the enclosed volume. We interpret this result as a natural extension of the "area law" for confinement that has long been established for chromodynamic strings. This result, in turn, paves the way to the introduction of fermion fields in the model and gives logical continuity to the calculations undertaken in this paper concerning the quantum properties of $A_{\mu \nu \rho}$.

This paper extends our previous results in two ways. First, we have calculated the contribution to the Casimir pressure due to the zero point oscillations of $A_{\mu \nu \rho}$ in the massive case. We find that the critical quantum effect due to the integration constant of the homogeneous massless equation, is still in place leading to the characteristic Casimir pressure that is inversely proportional to the volume of the bag. In addition, there is now a contribution to the vacuum pressure, Eq. (40), that is directly related to the mass of the $A$-field and plays the same role as the phenomenological bag constant introduced as an infrared cutoff in the partition functional for the massless case.

The second extension concerns the effect of the newly derived vacuum pressure on the effective dynamics of fermion fields. This amounts, in practice, to a recalculation of the Wilson loop extended to the full quantum case in which the classical current (49) is replaced by the spin-density current (50) of the fermion field. The result is displayed in Eq. (63) and is noteworthy for the explicit presence of the quantum propagator associated with the field equation (11) discussed in the Introduction.

Just as in the case of the confining static potential associated with a relativistic test bubble, the dipole structure of the quantum propagator in Eq. (63) in the present case is symptomatic of the confining and screening properties of the $A_{\mu \nu \rho}$ field at the quantum level.

We have repeatedly emphasized the stringent similarity between these properties of the $A$-field in four dimensions and those of the "electromagnetic" field in two space-time dimensions. The exact nature of this analogy and its possible 
impact on the problem of color confinement in QCD will be discussed in a forthcoming publication.

[1] S.Ansoldi, A.Aurilia, E.Spallucci Phys. Rev. D64, 025008 (2001)

[2] A.Aurilia, E.Spallucci Quantum fluctuations of a "constant" gauge field, hep-th/0402096 in print on Phys.Rev.D

[3] S.Hawking Phys. Lett. B 134, 403 (1984)

J.D.Brown, C.Teitelboim Nucl. Phys.B297, 787 (1985)

M. J. Duff Phys. Lett. B 226, 36 (1989)

M.J. Duncan, L.G. Jensen Nucl. Phys. B 336, 100 (1990)

N. Turok, S.Hawking Phys. Lett. B 432, 271 (1998)

[4] Y. Fujii, T. Inami, M. Kato and N. Ohta, Phys. Lett. 131B (1983) 319

N. Ohta, Phys. Lett. 138B (1984) 63,

N. Ohta, Zeit. für Phys.C26 (1985) 535,

[5] A.Aurilia, D.Christodoulou, F.Legovini Phys. Lett. B73, 429 (1978)

[6] Y.Kazama, R.Savit Phys.Rev.D21 2916 (1980)

P.Di Vecchia, G. Veneziano Nucl. Phys. 171253 (1980)

K. Kawarabayashi, N.Ohta Nucl. Phys. 175477 (1980)

E.Witten Ann. Phys.128 363 (1980)

A.Aurilia, H.Nicolai, P.K.Townsend Nucl. Phys. B 176, 509 (1980)

K. Kondo Progr. Theor. Phys. Suppl. 131243 (1998)

G. Gabadadze Phys. Rev. D58, 094015 (1998)

[7] A.Aurilia, Phys. Lett. B 81, 203 (1979)

[8] A.Aurilia, Y. Takahashi Progr. Theor. Phys. 66, 693 (1981)

N. Ohta, Progr. of Theor. Phys. 66 (1981) 1408

[9] P.K. Townsend Phys. Lett. 88B, 97 (1979)

M.Namazie, D.Storey J.Phys. A13, L161 (1980)

T.Kimura, Progr.Theor. Phys. 64, 357 (1980)

W.Siegel Phys. Lett. 93B, 170 (1980)

H.Hata, T.Kugo, Ohta Nucl. Phys. B178 527 (1981)

[10] S.Ansoldi, A.Aurilia, E.Spallucci Eur. J. Phys. 21, 1 (2000)

[11] M. Luscher Phys. Lett. B 90, 277 (1980)

[12] A.Aurilia, F.Legovini Phys. Lett.B 67, 299 (1977)

M.Henneaux, C.Teitelboim Found. of Phys. 16, n.7, 593 (1986)

S. Ansoldi, A. Aurilia, A. Smailagic, E. Spallucci Phys. Lett. B471, 133 (1999)

[13] K.Seo, A.Sugamoto Phys.Rev.D24 1630 (1981)

M.C. Diamantini Phys.Lett.B388 273 (1996)

M.C. Diamantini, F.Quevedo, C.A. Truegenberger Phys.Lett.B396 115 (1997)

F.Quevedo, C.A. Truegenberger Nucl. Phys. B 501, 143 (1997)

S. Ansoldi, A. Aurilia, L. Marinatto, E. Spallucci Prog.Theor.Phys.103 1021 (2000)

A.Smailagic, E.Spallucci Phys.Rev.D61 067701 (2000)

A.Smailagic, E.Spallucci Int.J.Phys.A 34435 (2001)

M.C. Diamantini Phys.Lett.B512 174 (2001)

H.Ruegg, M. Ruiz-Altaba The Stuckelberg field, hep-th/0304245 